\title{
Mobile Arbeit - räumlich entgrenzt und ortsgebunden
}

\author{
Anna Monz ${ }^{1}$ Gerlinde Vogl²
}

Online publiziert: 24. August 2020

(c) Der/die Autor(en) 2020

\section{Zusammenfassung}

Der Beitrag beschäftigt sich mit Anforderungen ortsgebundener mobiler Arbeit und den daraus resultierenden Belastungen. Die Diskussion um mobile Arbeit wird überwiegend vor dem Hintergrund zunehmender räumlicher und zeitlicher Gestaltungsspielräume von Wissensarbeiter/-innen geführt. In diesem Beitrag steht hingegen die ortsgebundene mobile Arbeit von Techniker/-innen im Fokus, welche die Anwesenheit der Beschäftigten bei Kund/-innen zwingend voraussetzt. Anhand der Empirie werden neue Anforderungen analysiert, die im Zusammenhang mit der Digitalisierung stehen und sich sowohl auf die Tätigkeit und den Arbeitsprozess selbst als auch auf die räumliche und digitale Mobilität auswirken.

Praktische Relevanz: Die empirischen Ergebnisse verweisen auf die Notwendigkeit einer Arbeitsgestaltung, die neben den Anforderungen, die sich aus der konkreten Arbeitstätigkeit ergeben, auch die mobilitätsbedingten Anforderungen mit in den Blick nimmt. Erst durch die systematische Analyse des Zusammenwirkens beider Bereiche ergeben sich spezifische Erkenntnisse für die Gestaltung von mobiler ortsgebundener Arbeit. Durch die Unterscheidung in arbeitsund mobilitätsbezogene Anforderungen wird zudem der Fokus auf die räumliche Mobilität und auf die dazu notwendige Mobilitätsarbeit gelenkt.

Schlüsselwörter Mobile Arbeit · Mobilität · Service- und Wartungstechniker · Digitalisierung

\section{Mobile work—spatially blurred and local fixed}

\begin{abstract}
This contribution focuses on job mobility requirements and the resulting strains on employees. The discussion about mobile work mostly focuses on the increase of spatial and temporal flexibility for knowledge workers. This contribution, by contrast, focuses on job mobility in locally fixed jobs, where the employee has to be present at the customers' place. The analysis of the empirical data shows that job mobility combined with locally fixed work activities implies special challenges for the employees. Moreover, the challenges have to be seen against the background of digitization. New challenges are found regarding both the working process/working activity itself and the organization of mobility.

Practical Relevance: Empirical data emphasizes the need for a work organization that systematically integrates the diverse challenges of job mobility requirements. An analysis of the interrelation between the demands emerging from the actual work-related tasks on the one hand and the demands emerging from mobility-related tasks on the other hand is necessary to get specific insights into the design of mobile work in locally fixed jobs. By distinguishing between work-related and mobility-related requirements, spatial mobility and the respective "mobility work" are focused.
\end{abstract}

Keywords Mobile work $\cdot$ Mobility $\cdot$ Service technicians $\cdot$ Digitization

Anna Monz

Anna.Monz@med.uni-muenchen.de

1 Institut für Sozialwissenschaftliche Forschung, Universitätsklinik München, München, Deutschland

2 Input Consulting, Stuttgart, Deutschland

\section{Einleitung}

Arbeit wird zunehmend mobil. Mobiles Arbeiten ist heute für viele Beschäftigte unterschiedlicher Qualifikationen und in fast allen Branchen zu einer Selbstverständlichkeit geworden. Immer mehr Arbeitsanteile werden durch die 
Nutzung mobiler Informations- und Kommunikationstechnologien ortsflexibel, d. h. können von jedem beliebigen Ort aus erledigt werden. Der stationäre Arbeitsort verliert an Bedeutung, jeder private oder öffentliche Raum wird zum potenziellen Arbeitsort.

Das „Raum-Zeit-Regime“ (van de Pol 2007) der Arbeit hat sich von der Industriegesellschaft zur Dienstleistungsoder Wissensgesellschaft deutlich verändert. Die Informatisierung (Schmiede 1996) von Wirtschaftsprozessen hat die Bindung der Arbeit an feste physische Orte erheblich gelockert und so die Möglichkeit geschaffen, dass immer mehr Arbeitstätigkeiten mobil ausgeübt werden können. Mobilität wird so für immer mehr Beschäftigte zu einem strukturierenden Element ihrer beruflichen Tätigkeit (Kesselring und Vogl 2010; Kesselring 2012) und ihres privaten Alltags (Monz 2018).

(...) the increasingly globalised nature of multinational corporations, combined with the evolution of laptop computers, mobile phones and internet-based means of communication, mean that spatial mobility for workers is potentially both more necessary and more possible(Hislop und Axtell 2007, S. 49).

Die räumliche und zeitliche Entgrenzung von Arbeit kann Beschäftigten auch neue Mitgestaltungsmöglichkeiten bei der Wahl des Arbeitsorts ermöglichen. Allerdings trifft dies in erster Linie auf hochqualifizierte Tätigkeiten zu, in diesem Segment verfügen Beschäftigte in der Regel über mehr Möglichkeiten zu örtlich und zeitlicher Flexibilisierung.

Anders stellt sich die Situation bei personenbezogenen Dienstleistungen oder im Handwerk dar: hier ist die körperliche Anwesenheit des Beschäftigten oftmals notwendig (Cohen 2010, Gressel et al. 2019). Die Beschäftigten müssen sich räumlich bewegen, um ihre Arbeit zu tun. Die Interaktion mit Kunden oder KollegInnen oder handwerkliche Arbeiten erfordern körperliche Anwesenheit an einem bestimmten Ort, sie sind ortsgebunden. Es handelt sich hier um ortsgebundene mobile Arbeit, die sich nicht remote erledigen lässt, sondern mit „temporal-spatial fixes“ (Jessop 2006) einhergeht. Diese Ortsgebundenheit mobiler Arbeit wird auch durch die Auswertung des European Working Conditions Survey bestätigt:

Anders als die öffentliche Debatte vermuten lässt, findet mobiles Arbeiten jedoch nur selten in den eigenen vier Wänden, im Café oder Freibad statt, sondern hauptsächlich beim Kunden (Hammermann und Stettes 2017, S. 3).

Frank Kleemann spricht von ,mobiler Präsenz-Arbeit“ (2017, S. 222) und meint damit die physisch gebundene Arbeit, die der Anwesenheit an einem konkreten Ort bedarf. So zeichnen sich die Arbeitstage der hier untersuchten Techniker/-innen dadurch aus, dass sie ihre Arbeitszeit teilweise direkt beim Kunden oder mit der An- und Abreise zum Kunden verbringen (Gressel und Munduteguy 2008). Mobilität ist eine notwendige Voraussetzung zur Erledigung der Arbeit. Sie ist Teil der im Rahmen des Arbeitsverhältnisses zu erbringenden Arbeitsleistung und somit „,verordnete Mobilität“ (Nies et al. 2015, 2017), die dadurch charakterisiert ist,

dass spezifische Anteile der Arbeit ,,außerbetrieblich ortsgebunden" sind und damit die räumliche Bewegung der jeweiligen Beschäftigten erfordern (Nies et al. 2017, S. 176).

Ortsgebundene mobile Arbeit; wird in der Debatte zu mobiler Arbeit kaum thematisiert (Cohen 2010; Nies et al. 2017; Gressel et al. 2019), was auf die Dimension der sozialen Ungleichheit von mobiler Arbeit verweist: Der Freiheitsdiskurs, der die Debatte um mobiles Arbeiten bestimmt, passt nicht auf alle Formen mobiler Arbeit und damit auch nicht auf alle Beschäftigten gleichermaßen.

Die spezifischen Anforderungen ortsgebundener, mobiler Arbeit unterscheiden sich von anderen Formen mobiler Arbeit. Zudem entstehen durch die Digitalisierung auch bei ortsgebundener, mobiler Arbeit neue Anforderungen an die Beschäftigten, wie wir im Folgenden zeigen werden.

\section{Methode und Sample}

Im Folgenden werden Resultate des Forschungsprojekts „prentimo - Präventionsorientierte Gestaltung mobiler Arbeit" vorgestellt, das sich auf die Arbeit beim Kunden als einer Form räumlicher Mobilität in der Arbeit konzentrierte. ${ }^{1}$

Zur Beantwortung der projektrelevanten Fragestellungen wurden unterschiedliche Methoden - Fragebögen (Kraus und Rieder 2018), Beobachtungsverfahren (Dunckel und Pleiss 2007) und qualitative Interviews (Witzel 1985) - verwendet. In diesem Beitrag werden die Ergebnisse der qualitativen Interviews dargestellt. Die Methode des qualitativen Interviews ermöglicht es, die subjektive Wahrnehmung und Deutung der Beschäftigten hinsichtlich ihrer Arbeit sowie die Perspektive von Expert/-innen in unterschiedlichen Funktionen auf die betrieblichen Rahmenbedingungen und Umgangsstrategien zu erheben (Flick et al. 2012; Bogner et al. 2014).

Es wurden qualitative, leitfadengestützte Interviews mit Techniker/-innen $(n=45)$ geführt. Der Fokus lag dabei auf der differenzierten Erfassung der arbeits- und mobilitätsbezogenen Anforderungen, auf der subjektiven Wahrnehmung ihres mobilen Arbeitsalltags, und des individuellen Arbeits-

\footnotetext{
${ }_{1}$ Weitere Informationen zum Forschungsprojekt prentimo: www. prentimo.de.
} 
handelns sowie auf Veränderungen des Arbeitsalltags durch die Digitalisierung.

Zudem wurden zur Erhebung der betrieblichen Rahmenbedingungen qualitative Experteninterviews $(n=12)$ mit betrieblichen Vertretern aus den Bereichen Personalmanagement, Betriebliches Gesundheitsmanagement, Arbeitsschutz und Interessenvertretung durchgeführt. Der inhaltliche Fokus lag dabei auf der Organisation mobiler Arbeit im Unternehmen, den Veränderungen von Anforderungen durch die Digitalisierung, den Belastungen der Beschäftigten sowie neuen technischen Entwicklungen und dadurch entstehenden neuen Herausforderungen für die Beschäftigten.

Die leitfadengestützten Interviews der Expert/-innen sowie der Beschäftigten dauerten jeweils zwischen 60 und 90 min. Sämtliche Interviews wurden aufgezeichnet, transkribiert und im Anschluss computergestuitzt mit der Software MAXQDA auf Grundlage der qualitativen, inhaltlich strukturierenden Inhaltsanalyse nach Kuckartz (2018) ausgewertet. Die Auswertung des empirischen Materials erfolgte im Projektteam.

Die Techniker/-innen, die wir im Rahmen des Projekts untersucht haben, sind bei einem Telekommunikationsunternehmen und in einem Unternehmen der Facility-Management Branche beschäftigt. Aus dem Telekommunikationsunternehmen wurden 28 Beschäftigte und neun Expert/-innen interviewt, in dem Facility-Management Unternehmen waren es 17 Beschäftigte und drei Expert/-innen. Alle Altersgruppen sind im Sample vertreten, wobei die Gruppe der über 50 jährigen Beschäftigten etwas überrepräsentiert ist. Unter den interviewten Techniker/-innen waren fünf Frauen und 40 Männer. Diese Zahlen spiegeln zunächst die Geschlechterdifferenz in den untersuchten Branchen und Tätigkeitsfeldern wider, in denen unabhängig von der Mobilität überwiegend männliche Beschäftigte zu finden sind. Sie verweisen aber auch auf einen Zusammenhang zwischen Geschlecht und mobiler Arbeit. Die Mobilität zwischen Männern und Frauen unterscheidet sich zwar nicht per se, wenn jedoch Kinder im Haushalt leben, sind Frauen selten beruflich mobil, während die Familiensituation bei Männern keinen Einfluss auf deren Mobilität nimmt (Rüger und Becker 2011).

Die Beschäftigten des Samples arbeiten im Laufe eines Arbeitstags meist an unterschiedlichen Standorten, die in einem räumlich definierten Gebiet liegen. Die zeitliche und räumliche Planung ihres Arbeitstags erfolgt zentral durch Disponent/-innen.

Mit Blick auf die Mobilitätsform lassen sich bei den hier untersuchten Beschäftigtengruppen zwei Formen unterscheiden: die Tages- und die Übernachtmobilität. Bei der Tagesmobilität sind die Beschäftigten innerhalb der Arbeitszeit auf kürzeren Strecken zwischen verschiedenen Kund/-innen mobil und kehren nach dem letzten Einsatz nach Hause zurück. Die Mehrzahl der interviewten Beschäftigten war tagesmobil, lediglich ein geringer Anteil war darüber hinaus mit Übernachtmobilität konfrontiert.

Bei der Tagesmobilität werden die Fahrten zum Kunden ausschließlich mit dem Dienstwagen (Pkw oder [Klein-] Transporter) unternommen. Die Techniker/-innen sind sowohl im urbanen als auch im ländlichen Raum unterwegs. Im urbanen Raum werden zwar weniger Kilometer pro Tag zurückgelegt, die Verkehrssituation ist jedoch stärker durch Behinderungen (Stau) sowie Parkplatzsuche geprägt. Im Folgenden steht die Tagesmobilität der Beschäftigten im Fokus der Ausführungen.

\section{Ergebnisse: (Neue) Anforderungen ortsgebundener mobiler Arbeit}

Die Mehrheit der interviewten Beschäftigten hat eine für die Ausübung ihrer Tätigkeit einschlägige Berufsausbildung absolviert. Die Aufgaben der Techniker/-innen im Telekommunikationsbereich umfasst beispielsweise die Inbetriebnahme von Telefonanschlüssen oder Netzwerklösungen und das Beheben von Netzstörungen für Privat- und Geschäftskund/-innen; im Facility-Management geht es in erster Linie um Wartung, Reparatur und den Einsatz in Notfällen, wie z.B. dem Ausfall einer Klimaanlage oder eines Wasserschadens.

Anforderungen ortsgebundener mobiler Arbeit zeigen sich dabei empirisch auf zwei Ebenen.

Zum einen gibt es die arbeitsbezogenen Anforderungen, die den Kern der Arbeitstätigkeit ausmachen, d.h. die technische Tätigkeit, der Kundendienst an der Maschine, sowie die Interaktionsarbeit mit den Kund/-innen (Dunkel und Rieder 2006), zum anderen die mobilitätsbezogenen Anforderungen, die die Organisation der Mobilität, den Umgang mit unvorhergesehenen und weitgehend unvorhersehbaren Mobilitätsereignissen sowie das damit verbundene Zeitma-

Tab. 1 Anforderungsebenen mobiler Arbeit (eigene Darstellung) Table 1 Levels of requirements for mobile work

\begin{tabular}{|c|c|}
\hline \multicolumn{2}{|l|}{ Anforderungen } \\
\hline $\begin{array}{l}\text { Arbeitsbezogen } \\
\text { Technische Tätigkeit }\end{array}$ & $\begin{array}{l}\text { Mobilitätsbezogen } \\
\text { Mobilitätsarbeit }\end{array}$ \\
\hline $\begin{array}{l}\text { Technisches Wissen und Kom- } \\
\text { petenzen } \\
\text { Arbeit bei und mit Kunden }\end{array}$ & $\begin{array}{l}\text { Räumliches (Erfahrungs-)Wis- } \\
\text { sen } \\
\text { Ortskenntnisse } \\
\text { Mobilitätskompetenzen }\end{array}$ \\
\hline \multicolumn{2}{|c|}{$\begin{array}{l}\text { Arbeits- und mobilitätsbezogene Anforderungen } \\
\text { Umgang mit unvorhersehbaren Situationen, situatives Handeln, } \\
\text { Selbststeuerung und -organisation, Einsatz und Austausch von fachli- } \\
\text { chem, interaktionsbezogenem und räumlichem Erfahrungswissen }\end{array}$} \\
\hline $\begin{array}{l}\text { Arbeitsorganisatorische Rahm } \\
\text { Gestaltung von Arbeitsprozesse } \\
\text { triebliche Organisation von Zei } \\
\text { und Reisezeit, Gestaltung von A }\end{array}$ & $\begin{array}{l}\text { dingungen: Leistungssteuerung, } \\
\text { Zuge der Digitalisierung, be- } \\
\text { l Raum (Regelung von Arbeits- } \\
\text { tsorten unterwegs) }\end{array}$ \\
\hline
\end{tabular}


nagement beinhalten. Weiter gibt es Anforderungen, die quer zu dieser Unterscheidung liegen und für beide Anforderungsbereiche gelten. Dazu gehören beispielsweise der Umgang mit unvorhersehbaren Situationen oder die Notwendigkeit situativen Handelns.

Tab. 1 verdeutlicht die verschiedenen Anforderungsebenen:

Für einen tieferen Einblick in die arbeits- und mobilitätsbezogenen Anforderungen, wird im Folgenden die ortsgebundene mobile Arbeit in die raum-zeitlichen Dimensionen des Vor-Ort-Seins, dem Unterwegs-Sein und dem Weg-Sein zerlegt. Die einzelnen Dimensionen und sozialen Zusammenhänge sind dabei nicht exakt voneinander $\mathrm{zu}$ treffen, sie bedingen sich vielmehr gegenseitig und nehmen aufeinander Einfluss. So bedeutet beispielsweise das „Vor-OrtSein“ gleichzeitig immer auch ein ,Weg-Sein“.

\subsection{Vor-Ort-Sein}

Die Techniker/-innen arbeiten überwiegend direkt beim Kunden. Das Vor-Ort-Sein bezieht sich auf die von der Betriebsstätte entfernten multiplen und flexiblen Arbeitsorte, die häufig dadurch charakterisiert sind, dass sie für die Arbeit der Techniker/-innen weder vorgesehen noch gestaltet sind.

Mein Arbeitsort ist dort, wo der Laptop gerade hinpasst. (Wartungstechniker Facility Management)

Durch die wechselnde Einsatztätigkeit der Techniker/-innen ändert sich die soziale als auch die räumliche Situation von Kund/-in zu Kund/-in. Die Techniker/-innen müssen sich immer wieder neu und flexibel auf variierende räumliche und soziale Situationen einstellen. Die Interaktionsarbeit, welche die Kommunikation mit Kund/-innen, das Aushandeln und die Bewältigung von unterschiedlichen Erwartungen und Konflikten umfasst, stellt neben der fachlichen Arbeit eine wichtige Anforderung dar. Diese wird häufig als Herausforderung erlebt, beispielsweise, wenn Kund/-innen Erwartungen haben, welche die Techniker/-innen im Rahmen ihres Auftrags nur schwer oder nicht erfüllen können, oder wenn es zu Konflikten bzw. zu Ärger über den Service des Unternehmens kommt.

Die Techniker/-innen sind in erster Linie allein unterwegs. Entscheidungen vor Ort müssen sie daher allein treffen. Das wird einerseits als positiv erlebt, da es mit einem hohen Anteil an Autonomie und Selbstbestimmung verbunden wird. Andererseits ist dieser Umstand eine Belastung aufgrund der hohen Verantwortung, die sie tragen. Als wichtig und hilfreich erleben die Techniker/-innen in diesem Zusammenhang einen gewissen, vom Unternehmen zugesicherten Handlungsspielraum, um eigenverantwortlich und situativ handeln zu können.
Neben der technischen und handwerklichen Tätigkeit gehört die Dokumentationsarbeit zu den täglichen Aufgaben der Techniker/-innen. Diese soll zeitnah nach Abschluss des jeweiligen Kundenauftrags erledigt werden. Im Zuge der Digitalisierung der Prozesse hat sie sich ebenfalls verändert: Sie soll nun direkt am Arbeitsort mithilfe von mobilen Endgeräten erledigt werden. Technische Hürden, wie z.B. eine schlechte Internetverbindung an den Arbeitsorten und Probleme bei dem Programm, das sie durch die Dokumentationsschritte führt, erleben die Beschäftigten als Belastung. Häufig kommt es dadurch zu Zeitverzögerungen und es entsteht hoher Zeitdruck.

Für die Erledigung der notwendigen Dokumentationsarbeiten ist bei den Kund/-innen meist kein Platz vorgesehen, die Beschäftigten führen diese Arbeit oft in provisorischen Räumen durch, die sie sich selbst suchen müssen. So arbeiten sie häufig an Durchgangsorten wie im Flur oder in Warteräumen, auf einer Bank oder anderen Sitzgelegenheit im öffentlichen Raum, im Auto oder auch zu Hause.

Die Arbeit zu Hause wird in der Debatte um mobile Arbeit meist als positive Option und Chance gerahmt. Für die Techniker/-innen ist die Erledigung der Dokumentationsarbeit zu Hause nur eine Notlösung. Durch die zunehmende Verlagerung der Dokumentationsarbeiten nach Hause sowie die Erreichbarkeit am Wohnort außerhalb der Arbeitszeiten verschwimmen die Grenzen zwischen Arbeit und Privatleben, was von den Techniker/-innen eher negativ bewertet wird.

Besteht die Möglichkeit, dass die Techniker/-innen Räume in den Kundenobjekten mitbenutzen, sind diese meist weder für Büroarbeit noch für Pausen oder gemeinsamen Austausch gut geeignet.

Es ist meistens eine Abstellkammer ohne Fenster, oder was weiß ich, ist ja auch nicht gerade toll. Reinigungssachen und Putzfolien, da sitzt man dann. (Wartungstechniker Facility Management)

Während Arbeitsräume oder Arbeitsplätze sonst überwiegend als permanente Orte gedacht werden und für die Ausübung der Arbeitstätigkeit konzeptualisiert, ein- und ausgerichtet sind, trifft dies für die Arbeitsorte mobil Arbeitender oft nicht $\mathrm{zu}$.

Fehlende Arbeitsräume führen auch dazu, dass mobil Beschäftigte ihre Pausen ausfallen lassen oder während der Autofahrt von einem Kunden zum anderen ihre Mahlzeit einnehmen. Zudem ist es oft schwierig, Hygieneeinrichtungen aufzusuchen, um sich z.B. die Hände zu waschen.

Wir haben jetzt auch keine Duschen oder auch keinen Pausenraum und auch eigentlich keine Mikrowelle (...) eigentlich gar nichts. (Wartungstechniker Facility-Management) 
Mobil Beschäftigte, die beim Kunden arbeiten, bewegen sich nicht nur in physischen, sondern auch in sozialen Räumen, die ihnen fremd sind und in denen sie sich zurechtfinden müssen. Anders als beim eigenen Arbeitgeber, wo man Kolleg/-innen und auch die Organisation und deren Abläufe kennt und sich in vertrauten sozialen Räumen bewegt, ist die Situation beim Kunden meist fremd. Hinzu kommt, dass Techniker/-innen sich selbst als störend für die Belegschaft des Kunden erleben. Häufig besteht ein soziales Gefälle zwischen ihnen, wie z.B. bei der Arbeit in Banken. Bei den Techniker/-innen entsteht die Wahrnehmung, ihre Arbeit im Verborgenen tun zu müssen und nicht auffallen zu dürfen. Die Techniker/-innen erleben diese Situation als einen räumlichen Ausdruck mangelnder Wertschätzung seitens des Kunden - eine räumliche Manifestation der Ungleichheit, die zwischen ihnen und der Belegschaft des Kunden besteht.

\subsection{Unterwegs-Sein}

Das Unterwegs-Sein betrifft die Bewegung im Raum selbst, also die Bewegung von einem Kunden zum nächsten. Die Hauptanforderung liegt hier im Umgang mit unterschiedlichen Verkehrssituationen bzw. der Bewältigung des Verkehrs. Der Verkehr ist dabei für die Techniker/-innen mit drei Hauptrisiken verbunden: Als erstes Hauptrisiko gilt der Stau (ob es sich nun um vorhersehbare und wiederkehrende Staus wie z.B. tageszeitenabhängige Verkehrsspitzen handelt oder um unvorhersehbare Verkehrsprobleme wie Staus infolge eines Unfalls). Das zweite Risiko stellen mangelnde Parkplätze am Einsatzort dar. In den Innenstädten ist der Parkdruck oftmals so hoch, dass die Techniker keinen Parkplatz in der Nähe ihres Einsatzorts finden, was dazu führt, dass sie das notwendige Material und das zum Teil schwere Werkzeug zum Teil weit tragen müssen. Das dritte Risiko sind Pannen oder Unfälle, in welche die Techniker verwickelt sind.

Mobilität ist für die Techniker/-innen vor allem ein Zeitfresser. Da sie einen straffen Zeitplan für die Erledigung ihrer Aufträge haben, stellen mobilitätsbedingte Verzögerungen eine zusätzliche Belastung dar. Die betriebliche Planung der Reisezeiten ist jedoch häufig knapp bemessen, da die Betriebe mobilitätstypische Verzögerungen in der Regel unzureichend in die Planung einbeziehen. Verspätungen und Zeitdruck gehören daher für Techniker/-innen zur Normalität ihres Arbeitsalltags. So können sie häufig vereinbarte Termine nicht einhalten und sehen sich in der Folge mit verärgerten Kund/-innen konfrontiert.

Hinzu kommt, dass die Vorgabezeiten für standardisierte Tätigkeiten reduziert wurden:

Und die Zeiten werden jährlich nach unten korrigiert. Schon seit Jahren. Ob das für eine Schaltung ist, an ei- nem Verteiler. Und die Zeiten sind oftmals eben nicht einzuhalten. (Servicetechniker Telekommunikation)

In der Folge kommt es zu einer moderaten, aber kontinuierlichen Arbeitszeitüberschreitung.

Arbeitsende, da gibt es im Grunde keine Definition. Meine Frau hat sich auch mittlerweile abgewöhnt, mal anzurufen und zu fragen: „Wann bist du denn zu Hause?" Das wissen wir nie. (Servicetechniker Telekommunikation)

Diese Situation konfrontiert Beschäftigte mit widersprüchlichen Anforderungen: dem Wunsch, die Arbeitsaufgabe beim Kunden zufriedenstellend zu lösen, dem eigenen Bedürfnis, die Arbeitszeit nicht zu überschreiten, und den Vorgaben des Unternehmens, sich an Zeitvorgaben zu halten und ausschließlich Arbeiten durchzuführen, die auch in Rechnung gestellt werden können.

Es wird jedoch nicht nur ein zunehmender Zeitdruck thematisiert, sondern auch der Verlust von Autonomie in der Mobilitätsgestaltung. Im Zuge der Digitalisierung und der damit verbundenen Standardisierung der Arbeitsabläufe hat auch eine Automatisierung der Routenplanung stattgefunden. Wo sich Techniker/-innen früher ihre Routen selbst zusammenstellten, wird ihnen die Route heute digital vorgegeben. Sie erleben das zum einen als Verlust ihrer Gestaltungsfreiheit, zum anderen als Entwertung ihres räumlichen Erfahrungswissens.

Häufig erscheint ihnen die digitalisierte Routenplanung zudem als wenig effizient, wenn geographisch nah beieinander liegende Aufträge nicht zusammengefasst, stauanfällige Routen oder Baustellen nicht berücksichtigt werden. Das Erfahrungswissen der Techniker/-innen ermöglicht ihnen, situationsbezogen Routen zu wählen, die Zeitersparnisse erlauben und situationsbezogene Anforderungen zu berücksichtigen.

Auch wenn die Anforderungen an räumliche Flexibilität der mobilen Arbeit bis zu einem gewissen Grad inhärent sind, tragen betriebliche Praktiken zur Be- oder Entlastung der Beschäftigten bei. So berichten die Techniker/ -innen, dass betriebliche Büroräume mit dem Verweis auf die Möglichkeit mobilen Arbeitens aufgelöst werden. Für viele Techniker/-innen wird dadurch das Auto zum zentralen Arbeitsort.

Der Firmenwagen ist sowieso alles - Büro, Frühstücksort. Normalerweise muss man auch noch im Auto einen Campingstuhl und Campingtisch haben, wo man alles drauf stellt dann, für den Rechner, für die Protokolle. Das geht an sich nur noch im Auto. (Servicetechniker Telekommunikation)

Der Dienstwagen ist aber fürs Fahren, nicht aber für die Arbeit am Laptop vorgesehen und eingerichtet. Er ist kein 
Arbeitsplatz, tatsächlich dient er jedoch als Büro, als Material- und Ersatzteillager sowie zugleich als privater Raum und Erholungsort.

\subsection{Weg-Sein}

Die Dimension des Weg-Sein bezieht sich auf die Folgen der räumlichen Entfernung von Kolleg/-innen und Betriebsstätte. Eine damit verbundene Anforderung ist das isolierte Arbeiten unter Einschränkung des Kontakts und Austauschs mit Kolleg/-innen. Anders als bei stationärem Arbeiten in einem gemeinsamen Büro ergeben sich bei diesem mobilen Arbeiten keine Gelegenheiten zum absichtslosen Treffen von Kolleg/-innen und Face-to-Face-Interaktionen können nicht ad hoc hergestellt werden. Dennoch sind auch und gerade mobil Beschäftigte auf die Unterstützung und den Austausch untereinander angewiesen z. B. wenn sie beim Kunden vor Ort mit einem Problem konfrontiert werden, das sie nicht allein lösen können. Hier benötigen sie Unterstützung von Kolleg/-innen, die über das fehlende Wissen verfügen. Der Austausch informellen Wissens über Geräte, Anlagen, Problemlösestrategien oder Zugänge zu Gebäuden ist eine wichtige Ressource für die Bewältigung der Arbeitsanforderungen.

Die meisten Techniker/-innen starten ihren Arbeitstag nicht von der Betriebsstätte, sondern von zu Hause aus. Dies hat den Vorteil, dass sie morgens direkt zum ersten Kunden fahren können und nicht erst den Unternehmensstandort ansteuern müssen. Dieser ,optimierte Dienstantritt“ hat aber auch zur Folge, dass man sich nicht mehr automatisch über den Weg läuft, was den kollegialen Austausch erschwert. Die Techniker/-innen sind auf sich selbst gestellt, sie fühlen sich als ,Einzelkämpfer“.

Dass man sich sonst noch mal trifft wie früher, das ist nicht mehr gewollt und wird auch immer weniger. Man ist wirklich eigentlich ein Alleinkämpfer. Dann und wann trifft man natürlich mal einen Kollegen in den Technikräumen oder irgendwo. Aber ansonsten ist man durchaus überwiegend auf sich allein gestellt.

(Servicetechniker Telekommunikation)

Um den kollegialen Austausch sowie die gegenseitige Unterstützung dennoch zu gewährleisten, organisieren sie ihre Kommunikation in erster Linie virtuell. Häufiges Telefonieren und Chatten mit Kolleg/-innen gehört zum Arbeitsalltag und ersetzt spontane und informelle Face-to-FaceGespräche. Die hohe Bedeutung kollegialer Unterstützung in schwierigen Kundensituationen bringt es mit sich, dass die mobil Beschäftigten für ihre Kolleg/-innen auch außerhalb ihrer Arbeitszeit erreichbar sind. Das Wissen um die wechselseitige Erreichbarkeit wirkt entlastend, auch wenn der Preis dafür die Aufopferung der eigenen Freizeit ist.
Ich habe auch schon nachts um drei Uhr einen Kollegen raustelefoniert, weil ich nicht weiterkam, und wenn wir Kollegen untereinander anrufen, das funktioniert auch wunderbar. Weil, wenn wir uns nicht helfen, wer sonst? Von daher habe ich da auch kein Problem mit, ich fahre dann auch raus. (Wartungstechniker Facility Management)

Erreichbarkeit wird als selbstverständlich, aber auch als notwendige Voraussetzung für die Bewältigung der eigenen Arbeit erachtet. Wesentlich ist die Reziprozität dieser informellen Verabredung: Wenn man für seine Kolleg/-innen jederzeit erreichbar ist, kann man das im umgekehrten Fall gleichfalls von ihnen erwarten. Die wechselseitige Erreichbarkeit außerhalb der Arbeitszeit wird vor diesem Hintergrund als Normalität und nicht als Problem gerahmt. Diese informell organisierte Erreichbarkeit außerhalb der Arbeitszeit ist nicht betrieblich angeordnet, sondern basiert auf einem implizit geteilten Kollegialitäts- und Solidaritätsverständnis. Auf diese Weise bilden sich Arbeitskollektive auch über räumliche Distanzen hinweg, die medienvermittelt funktionieren.

Nicht nur die Beschäftigten, auch das Unternehmen profitiert von dieser informell verabredeten Erreichbarkeit: Durch das informelle Handeln der Beschäftigten werden die Funktionalität und der Erfolg des Services in Störfällen sichergestellt. Die Stabilität dieses Handelns fußt auf der hoch anspruchsvollen Situation, in der sich die Techniker/-innen tagtäglich befinden: alleine beim Kunden, konfrontiert mit einer unbekannten oder unvorhergesehenen technischen oder sozialen Herausforderung, deren Lösung situativ und spontan gefunden werden muss. Der regelmäßige Austausch von Erfahrungswissen hat in diesem Kontext für die Techniker/-innen eine hohe Bedeutung und stellt eine wichtige Ressource dar. Dennoch wird das Aufrechterhalten kollegialer Beziehungen von betrieblicher Seite kaum unterstützt. Treffen der Techniker/-innen werden von Vorgesetzten oftmals nicht gerne gesehen, da sie als unproduktive Zeiten gelten.

Mit der digitalisierten Routenplanung und der Ausrüstung der Techniker/-innen mit mobilen Endgeräten wurde auch die räumliche Ortung der Techniker/-innen ermöglicht. Auch wenn eine individuelle Auswertung der Standorte aufgrund einer Betriebsvereinbarung nicht zulässig ist, fühlen sich die Techniker/-innen dennoch kontrolliert. Das hat direkte Auswirkungen auf die Handlungsebene und damit auf den kollegialen Austausch, da sie trotz des Trackingverbots unsicher sind, ob sie von ihrer Route abweichen können um z.B. Kolleg/-innen zu unterstützen oder sich auszutauschen. 


\section{Diskussion der Ergebnisse}

Die Techniker/-innen sind in ihrem Arbeitsalltag mit unterschiedlichen Anforderungen konfrontiert: Sie lösen technische Probleme und führen Wartungsarbeiten durch, sie bewältigen die Interaktionsarbeit bei Kund/-innen vor Ort, sie organisieren ihre Mobilität und managen ihre Erreichbarkeit sowie die virtuelle Kommunikation mit Kund/-innen, Vorgesetzten und Kolleg/-innen. Die Lösung technischer Probleme und die Befriedigung von Kundenwünschen verstehen sowohl sie selbst als auch das Unternehmen als ihre Kernaufgaben. Die Anforderungen, die durch die räumliche Mobilität entstehen, werden dabei häufig marginalisiert.

Mobilität in der Arbeit wird von den Beschäftigten aber auch als ein Stück Freiheit definiert. Die Möglichkeit, in einem - wenn auch engen - vorgegebenen Rahmen Handlungs- und Gestaltungsautonomie bei der Erledigung ihrer Arbeitsaufgaben zu haben, erleben sie als positiv.

Die räumliche Mobilität der Techniker/-innen stellt eine Grundvoraussetzung zur Erfüllung ihrer Arbeitstätigkeit dar. Die daraus entstehenden Anforderungen beinhalten die Organisation der Mobilität, den Umgang mit unvorhergesehenen und weitgehend unvorhersehbaren Mobilitätsereignissen sowie das damit verbundene Zeitmanagement. Beschäftigte brauchen dazu Kompetenzen, um den Anforderungen auf den unterschiedlichen Ebenen gerecht zu werden. Anforderungen, die sich aus dem Mobilsein ergeben, definieren wir in Anlehnung an Günter Voß (2010), als Mobilitätsarbeit, worunter er das ,,aktive Herstellung von Stabilitäten und Bindungen als Voraussetzungen oder zur Folgenbewältigung für die Mobilisierung" versteht (Voß 2010, S. 121) Diese Mobilitätsarbeit wird auf betrieblicher Ebene häufig übersehen, nicht thematisiert und folglich sowohl in der Planung als auch bei der Gestaltung von Arbeitsprozessen nicht oder nur unzureichend berücksichtigt (Gressel 2018; Gressel et al. 2019). Folge davon ist, dass Anforderungen individualisiert und auf individueller Ebene bewältigt werden. Von den Beschäftigten werden Mobilitätsfähigkeit und -kompetenz (Kaufmann und Jemelin 2004) erwartet, die sie individuell entwickeln müssen und die vom Unternehmen nicht explizit als besondere Leistungen oder Kompetenzen anerkannt werden.

Mobilitätsbezogene auf der einen und tätigkeitsbezogene Anforderungen auf der anderen Seite können in Konflikt miteinander geraten, wie z.B. der hohe Zeitaufwand für die Mobilität und die zeitlichen Anforderungen beim Kunden. Diese zu vereinbaren wird häufig als Herausforderung erlebt. Die Bewältigung divergenter Anforderungen muss dabei nicht per se zu Überlastungen führen. Vielmehr ist deren Ausbalancieren ein akzeptierter Bestandteil qualifizierter Tätigkeiten und kann zu einer hohen Arbeitszufriedenheit führen (Moldaschl 2007, S. 299). Das ist vor allem dann der Fall, wenn die betrieblichen Rahmenbe- dingungen und die zur Verfügung gestellten Ressourcen es den Beschäftigten ermöglichen, die alltäglichen tätigkeits- und mobilitätsbezogenen Anforderungen miteinander zu vereinbaren und erfolgreich zu bewältigen. Fehlt dieser Handlungsspielraum, werden die widersprüchlichen Anforderungen als Überforderung erlebt.

Wesentliche Belastungen bei mobiler Arbeit werden durch neue Formen der Leistungssteuerung (Nies et al. 2017), zunehmende Erreichbarkeitserwartungen außerhalb der Arbeitszeit (Menz et al. 2016) sowie durch eine Entgrenzung und ein Eindringen der Arbeit in das Privatleben (Monz 2018) verursacht. So zeigen sich diese Dimensionen ebenfalls bei den Techniker/-innen als wichtige Stellschrauben bei der Frage, unter welchen Bedingungen mobile Arbeit als Bereicherung oder als Belastung erlebt wird (Rieder et al. 2019, 2020).

Zusätzlich erweist sich bei den Techniker/-innen die im Zuge der Digitalisierung voranschreitende Formalisierung und Standardisierung der Arbeitsprozesse als zentrale Rahmenbedingung für das Organisieren ihrer Tätigkeiten. Die Tendenz zu einer Standardisierung zeigt sich sowohl bei der Arbeitstätigkeit selbst als auch bei der Mobilität.

Durch die Digitalisierung und damit einhergehenden Standardisierung von Arbeitsabläufen werden einzelne Arbeitsschritte zwingend vorgegeben. Die Techniker/-innen verlieren dadurch Handlungsautonomie auf der inhaltlichen Ebene, d.h. in Bezug auf die Frage, wie ein technisches Problem zu lösen ist. Ihre Fachexpertise und ihr Erfahrungswissen werden entwertet; die hohe berufliche Identifikation und ihr Selbstverständnis als technische Problemlöser leiden darunter.

Im Bereich der Mobilität zeigt sich die Standardisierung in der Automatisierung der Routenplanung: Digitalisierte Dispositionssysteme geben die Reihenfolge der anzufahrenden Kunden vor, die Techniker/-innen können ihre Route, d.h. die Reihenfolge, in der sie die einzelnen Kunden anfahren, nicht mehr selbst planen und organisieren. Damit geht hinsichtlich der Routenplanung die Handlungsautonomie, hier auf einer räumlichen Ebene, verloren. Damit können sie ihre persönlichen Ortskenntnissen und Erfahrungen nicht mehr nutzen, um eventuellen Verkehrsstörungen auszuweichen und die vielen Unwägbarkeiten, die mit ihrer mobilen Arbeit verbunden sind, so weit wie möglich zu steuern, um dem Gefühl des Ausgeliefertseins zu entgehen.

Die Einführung von Ortungssystemen in den Autos fördern bei den Techniker/-innen das Gefühl des Kontrolliertwerdens. Diese Entwicklungen haben direkte Auswirkungen auf die Handlungsebene. Die Techniker/-innen können beispielsweise ihre Routen nicht mehr so planen, dass sie unterwegs Kolleg/-innen treffen. Während sie bislang auf informellem Wege die vorgegebene Route verlassen konnten, um Kolleg/-innen zu unterstützen, sich auszutauschen 
oder Orte zum Pausieren anzufahren, bleiben sie nun auf ihrer Route, weil sie befürchten, getrackt zu werden.

Die zunehmende Standardisierung der Auftragsbearbeitung im Zuge der Digitalisierung hat dazu geführt, dass der Arbeitstag und das Arbeitshandeln der Techniker/-innen immer transparenter und damit kontrollierbarer werden. Spielräume für informelles Handeln - das für die Erledigung ihrer Arbeit zentral ist - werden im Rahmen dieser Entwicklungen immer enger (vgl. hierzu auch Butollo et al. 2018, die darauf verweisen, dass der Weg zur Industrie 4.0 den Handlungsspielraum der Beschäftigten beschränken und ein Mehr an Standardisierung und Fremdsteuerung mit sich bringen kann). Zudem hat die räumliche Entkopplung betrieblicher Arbeitsplätze bei gleichzeitiger digitaler Anbindung den Zugriff des Unternehmens auf vormals privat klassifizierte Orte erweitert.

Wie Kleemann und Matuschek (2018) konnten auch wir beobachten, wie wichtig Spielräume für informelles Handeln der Arbeitenden als funktional notwendiger Teil von modernen ,,autonomen“, „vernetzten“ und „selbstlernenden" technischen Organisationssystemen sind, um die Arbeit der Techniker/-innen tatsächlich zu unterstützen, statt sie noch weiter zu komplizieren. Die digitale Auftragssteuerung nebst automatisierter Routenplanung scheint die Handlungsspielräume und das Erfahrungswissen der Techniker/-innen eher als Störfaktor zu betrachten denn als notwendige Ressource.

Um widersprüchlichen Anforderungen im Arbeitsalltag gerecht zu werden, mobilisieren Beschäftigte nicht nur beruflich-technische, sondern auch räumliche, soziale, private und informelle Ressourcen. Kollegialität oder die intrinsische Motivation der Techniker/-innen, die eigene Arbeit gut zu erledigen, werden von den Betrieben funktionalisiert und betrieblich nutzbar gemacht. Soziale Ressourcen zur Bewältigung von Belastungen stehen nicht nur den Arbeitenden zur Verfügung, sondern werden auch unternehmensseitig genutzt (z.B. um mit einem knappen Personalbestand auszukommen). Die wechselseitige Hilfeleistung wird damit von der freiwilligen, selbstbestimmten Aktivität zu einem normativ gerahmten Leistungserfordernis und verliert sowohl für den einzelnen Beschäftigten als auch für die Gruppe den entlastenden Charakter (Moldaschl 2007, S. 296).

Zudem verlagert der Einsatz informeller Ressourcen betriebliche Verantwortlichkeiten wie den Gesundheitsschutz auf die individuelle Ebene. Die ergonomische Gestaltung des Arbeitsplatzes, Bedarfe an fachlicher und sozialer Unterstützung am Arbeitsplatz sowie das Einhalten der Ruhe- und Pausenzeiten werden den Beschäftigten überlassen. Diese Wahrnehmung haben sich auch die Techniker/-innen selbst angeeignet. Stress und Überlastung erleben sie oftmals als selbstverschuldet. Insbesondere im Hinblick auf ihre Arbeitszeiten und ihre Erreichbarkeit außerhalb dieser
Zeiten sehen die Techniker/-innen sich selbst in der Verantwortung.

Der vielseitige Einsatz sozialer, informeller und privater Ressourcen ist ebenfalls im Zusammenhang mit Formen ergebnisorientierter Leistungspolitik und indirekter Steuerung zu sehen, die mit ,interessierter Selbstgefährdung“ (Peters 2011; Krause et al. 2012) einhergehen kann. Mit diesem Konzept wird das Phänomen gefasst, dass Beschäftigte vor dem Hintergrund der Erfolgsorientierung wissentlich nicht nur ihre Gesundheit, sondern ihre gesamte Lebensbalance gefährden. Interessierte Selbstgefährdung zeigt sich z.B. in dem Phänomen des Präsentismus, dem Verzicht auf Pausenzeiten sowie der Missachtung von Arbeitszeitgrenzen. Studien haben gezeigt, dass dieses selbstgefährdende Verhalten vor allem als Folge indirekter Steuerung auftritt (Chevalier und Kaluza 2015). Kontrollformen, die sich am Ergebnis und Erfolg und nicht an der aufgewandten Arbeitskraft orientieren, verschleiern auch den Mehraufwand, der mobilitätsbedingt entsteht.

Unabhängig von der Ergebnisorientierung der Leistungspolitik identifizieren sich die Techniker/-innen stark mit ihrer Arbeit und fühlen sich ihren Kolleg/-innen und Kund/ -innen verpflichtet. Sie wollen ihre Arbeit gut machen, sowohl bezogen auf ihre eigenen arbeitsinhaltlichen Ansprüche (Nies 2015) als auch hinsichtlich der Zufriedenheit der Kund/-innen. Dafür nehmen die Techniker/-innen den Einsatz ihrer gesamten Ressourcen und die damit verbundenen Gefahren für ihr Privatleben - Gefahren, die durch veränderte Arbeits- und Mobilitätsbedingungen noch größer werden - in Kauf.

Die Dimension sozialer Ungleichheit spielt, so zeigen die Ergebnisse, auch in der mobilen Arbeit eine wichtige Rolle. Dies wird z.B. auf räumlicher Ebene sichtbar: Die Techniker/-innen erleben ihre Anwesenheit bei Kund/-innen als störend, und ihnen werden Räume zugewiesen, die ihre soziale Position symbolisieren. Im Gegensatz zu Wissensarbeiter/-innen, die beim Kunden sozial ,auf Augenhöhe“ arbeiten, verrichten die Techniker/-innen ihre Arbeit überwiegend ,im Verborgenen“. Ihre Arbeit soll im besten Falle nicht wahrgenommen werden; wird sie wahrgenommen, so wird dies eher als störend erlebt.

Bourdieu beschreibt den sozialen Raum als soziale Positionierung in Abhängigkeit von sozialem und ökonomischem Kapital und verweist damit auf den Zusammenhang von Macht, Herrschaft und sozialer Ungleichheit. Physische Räume sind damit immer auch soziale Räume, in denen sich Herrschaft ausdrückt, wie das folgende Zitat verdeutlicht:

Tatsächlich steht einem nichts ferner und ist nichts weniger tolerierbar als Menschen, die sozial fern stehen, aber mit denen man in räumlichen Kontakt kommt. (Bourdieu 1991, S. 32) 
Für die Techniker/-innen ist die erlebte Hierarchisierung des Raums ein symbolischer Ausdruck für die Hierarchie, die zwischen ihnen und der Belegschaft des Unternehmens besteht, in dem sie vorübergehend arbeiten.

Die empirischen Ergebnisse verdeutlichen die Notwendigkeit einer Arbeitsgestaltung, welche nicht nur die ,eigentliche" Arbeitstätigkeit, sondern auch die dafür notwendige Mobilitätsarbeit berücksichtigt. Erst durch die systematische Analyse des Zusammenwirkens beider Bereiche ergeben sich spezifische Erkenntnisse für die Gestaltung von mobiler Arbeit - und möglicherweise auch darüber hinaus. Weil es viele andere Berufsgruppen gibt, die in ähnlicher Form mobil sind, lassen sich die empirischen Ergebnisse über das hier vorgestellte Sample hinaus verallgemeinern.

Mobilisierung und Digitalisierung verändert nicht nur die Wissensarbeit, sondern auch das ortsgebundene oder ortsbezogene Arbeiten im Handwerk und insbesondere bei den personenbezogenen Dienstleistungen, die mobile Pflege sei hier als Beispiel angeführt. Die Auswirkungen auf die Tätigkeiten im Arbeitsalltag und die Organisation der Arbeit unterscheiden sich jedoch. Zukünftige Forschung sollte daher die Auswirkungen und Belastungen von räumlicher und digitaler Mobilität auf spezifische Tätigkeitsfelder differenzierter in den Blick nehmen.

Funding Open Access funding provided by Projekt DEAL.

Open Access Dieser Artikel wird unter der Creative Commons Namensnennung 4.0 International Lizenz veröffentlicht, welche die Nutzung, Vervielfältigung, Bearbeitung, Verbreitung und Wiedergabe in jeglichem Medium und Format erlaubt, sofern Sie den/die ursprünglichen Autor(en) und die Quelle ordnungsgemäß nennen, einen Link zur Creative Commons Lizenz beifügen und angeben, ob Änderungen vorgenommen wurden.

Die in diesem Artikel enthaltenen Bilder und sonstiges Drittmaterial unterliegen ebenfalls der genannten Creative Commons Lizenz, sofern sich aus der Abbildungslegende nichts anderes ergibt. Sofern das betreffende Material nicht unter der genannten Creative Commons Lizenz steht und die betreffende Handlung nicht nach gesetzlichen Vorschriften erlaubt ist, ist für die oben aufgeführten Weiterverwendungen des Materials die Einwilligung des jeweiligen Rechteinhabers einzuholen.

Weitere Details zur Lizenz entnehmen Sie bitte der Lizenzinformation auf http://creativecommons.org/licenses/by/4.0/deed.de.

\section{Literatur}

Bogner A, Littig B, Menz W (2014) Interviews mit Experten. Eine praxisorientierte Einführung. Springer VS, Wiesbaden

Bourdieu P (1991) Physischer, sozialer und angeeigneter physischer Raum. In: Wentz M (Hrsg) Stadt-Räume. Campus, Frankfurt a.M., New York, S 25-34

Butollo F, Jürgens U, Krzywdzinski M (2018) Von Lean Production zur Industrie 4.0. Mehr Autonomie für die Beschäftigten? http:// www.ais-studien.de/home/

veroeffentlichungen-18/oktober.html. Zugegriffen: 24. März 2020 (AIS 11(2):75-90)
Chevalier A, Kaluza G (2015) Psychosozialer Stress am Arbeitsplatz: Indirekte Unternehmenssteuerung, selbstgefährdendes Verhalten und die Folgen für die Gesundheit. Gesundheitsmonitor. Newsletter der Bertelsmann Stiftung und der BARMER GEK, Bd. 1, S $1-12$

Cohen RL (2010) Rethinking "mobile work": boundaries of space, time and social relation in the working lives of mobile hairstylists. Work, Employment and Society 24(1):65-84

Dunckel H, Pleiss C (2007) Kontrastive Aufgabenanalyse. Grundlagen, Entwicklungen und Anwendungserfahrungen. vdf, Zürich

Dunkel W, Rieder K (2006) Interaktionsarbeit und Koproduktion als Ressourcen für Innovationen. In: Streich D, Wahl D (Hrsg) Moderne Dienstleistungen. Impulse für Innovation, Wachstum und Beschäftigung. Beiträge der 6. Dienstleistungstagung des BMBF. Campus, Frankfurt a.M., New York, S 279-285

Flick U, von Kardorff E, Keupp H, von Rosenstiel L, Wolff S (Hrsg) (2012) Handbuch qualitative Sozialforschung. Beltz, Weinheim

Gressel R (2018) Une conflictualité difficile à faire émerger: Le cas des professionnels mobiles. Communication pour les JIST 2018, Paris, 9.-11. Juli 2018. Unveröffentlichtes Manuskript

Gressel R, Munduteguy C (2008) Les professionnels mobiles, Un groupe hétérogène avec une exposition importante au risque routier. RTS 99:147-167

Gressel R, Monz A, Vogl G (2019) Zur Ortsgebundenheit mobiler Arbeit. AIS 1:86-102 (online verfügbar unter: http://www.aisstudien.de)

Hammermann A, Stettes O (2017) Mobiles Arbeiten in Deutschland und Europa: Eine Auswertung auf Basis des European Working Conditions Survey 2015. Vierteljahresschr Empiri Wirtschaftsforsch 44(3): 1-23

Hislop D, Axtell C (2007) The neglect of spatial mobility in contemporary studies of work: The case of telework. New Technol Work Employ 22(1):34-51

Jessop B (2006) Spatial Fixes, Temporal Fixes, and Spatio-Temporal Fixes. https://www.researchgate.net/profile/Bob_Jessop3/ publication/253616218_Spatial_Fixes_Temporal_Fixes_and_ Spatio-Temporal_Fixes/links/00b495398433e7b7e4000000.pdf. Zugegriffen: 24. März 2020

Kaufmann V, Jemelin C (2004) La motilité, une forme de capital permettant d'éviter les irréversibilités socio-spatiales? Colloque de géographie sociale, Espaces et Sociétés aujourd'hui, Rennes

Kesselring S (2012) Betriebliche Mobilitätsregime. Zur sozio-geografischen Strukturierung mobiler Arbeit. Z Soziol 41(2):83-100

Kesselring S, Vogl G (2010) Betriebliche Mobilitätsregime. edition sigma, Berlin

Kleemann F (2017) Mobile und ortsungebundene Arbeit. In: HirschKreinsen H, Minssen H (Hrsg) Lexikon der Arbeits- und Industriesoziologie. Nomos, Baden-Baden, S 222-224

Kleemann F, Matuschek I (2018) Mensch und Technik revisited - Zum sich verändernden Stellenwert von Informalität im Prozess der Digitalisierung. http://www.ais-studien.de/home/veroeffentlichungen18/oktober.html. Zugegriffen: 24. März 2020 (AIS 11(2) :58-74)

Kraus S, Rieder K (2018) Fragebogen zur Analyse mobiler Arbeit - Amobil. Hochschule Aalen, Aalen. https://www.prentimo.de/ fragebogen-amobil. Zugegriffen: 24. März 2020

Krause A, Dorsemagen C, Stadlinger J, Baeriswyl S (2012) Indirekte Steuerung und interessierte Selbstgefährdung: Ergebnisse aus Befragungen und Fallstudien. Konsequenzen für das Betriebliche Gesundheitsmanagement. In: Badura B, Ducki A, Schröder H, Klose J, Meyer M (Hrsg) Fehlzeiten-Report 2012. Springer, Berlin, Heidelberg, New York, S 191-202

Kuckartz U (2018) Qualitative Inhaltsanalyse. Methoden, Praxis, Computerunterstützung, 4. Aufl. Beltz Juventa, Weinheim

Menz W, Pauls N, Pangert B (2016) Arbeitsbezogene erweiterte Erreichbarkeit. Wirtschaftspsychologie 18(2):55-66

Moldaschl M (2007) Ressourcenorientierte Analyse von Belastung und Bewältigung in der Arbeit. In: Moldaschl M (Hrsg) Immaterielle Ressourcen. Nachhaltigkeit von Unternehmensführung und 
Arbeit I. Arbeit, Innovation und Nachhaltigkeit, 2. Aufl. Hampp, München, Mering, S 285-322

Monz A (2018) Mobile Arbeit, mobile Eltern. Körperliche und virtuelle Kopräsenz in der Paarbeziehung berufsmobiler Eltern. Springer VS, Wiesbaden

Nies S (2015) Nützlichkeit und Nutzung von Arbeit. Beschäftigte im Konflikt zwischen Unternehmenszielen und eigenen Ansprüchen. edition sigma, Baden-Baden

Nies S, Roller K, Vogl G (2015) Räumliche Mobilität rund um die Arbeit. http://www.boeckler.de/pdf/p_fofoe_WP_001_2015.pdf. Zugegriffen: 2. Dez. 2019

Nies S, Roller K, Vogl G (2017) Mobilität und Leistung. Dienstreisende im Trade-off zwischen Verdichtung und Entgrenzung. Arbeit 26(2):173-191

Peters K (2011) Indirekte Steuerung und interessierte Selbstgefährdung: Eine 180-Grad-Wende bei der betrieblichen Gesundheitsförderung. In: Kratzer N, Dunkel W, Becker K, Hinrichs S (Hrsg) Arbeit und Gesundheit im Konflikt. Analysen und Ansätze für ein partizipatives Gesundheitsmanagement. edition sigma, Berlin, S $105-122$

Rieder K, Kraus S, Vogl G (2019) Mobile Arbeit: Arbeitsbedingungen und Erleben. In: Badura B, Ducki A, Schröder H, Klose J, Meyer M (Hrsg) Fehlzeitenreport 2019. Digitalisierung. Springer, Berlin, Heidelberg, New York, S 205-216
Rieder K, Kraus S, Vogl G (2020) On the road again: Wie kann die Arbeitsgestaltung zur Arbeitsfreude bei mobiler Arbeit beitragen? Organisationsberat Superv Coach 27:21-36. https://doi.org/10. 1007/s11613-020-00635-x

Rüger H, Becker K (2011) Berufsmobilität, Geschlecht und Lebensform. Berufsbedingte räumliche Mobilität und die Folgen für die Vereinbarkeit von Familie und Beruf und partnerschaftliche Arbeitsteilung. In: Klammer U, Motz M (Hrsg) Neue Wege - Gleiche Chancen. VS, Wiesbaden, S 363-396

Schmiede R (1996) Informatisierung, Formalisierung und kapitalistische Produktionsweise: Entstehung der Informationstechnik und Wandel der gesellschaftlichen Arbeit. edition sigma, Berlin

van de Pol R (2007) Einfluss der Informations- und Kommunikationstechnologien auf die zeitliche und räumliche Organisation der Arbeit: Universität Zürich. http://socio.ch/arbeit/t_vandepol.pdf. Zugegriffen: 20. Mai 2020

Voß G (2010) Mobilisierung und Subjektivierung. Und: Was würde Odysseus zum Thema Mobilität beitragen? In: Goetz I, Lehnert K, Lemberger B, Schondelmayer S (Hrsg) Mobilität und Mobilisierung. Campus, Frankfurt a.M., New York, S 95-136

Witzel A (1985) Das problemzentrierte Interview. In: Jüttemann G (Hrsg) Qualitative Forschung in der Psychologie. Grundfragen, Verfahrensweisen, Anwendungsfelder, S 227-255 